\title{
Formation des enseignants d'informatique au Maroc : Un regard critique
}

\section{Training of computer science teachers in Morocco: A critical review}

\author{
Radouane IQDOUR ${ }^{1 *}$, Ouafae $\mathrm{SERRAR}^{1}$, Oum El kheir $\mathrm{ABRA}^{2}$ \\ ${ }^{1}$ Centre Régional des Métiers de l'Education et de la Formation Marrakech-Safi, Maroc \\ ${ }^{2}$ Centre Régional des Métiers de l'Education et de la Formation Rabat-Kénitra, Maroc
}

\begin{abstract}
Résumé. L'introduction de l'enseignement de l'informatique en tant que science reste un sujet d'actualité dans plusieurs pays. Les principales questions qui surgissent à chaque fois concernent le curricula de l'informatique, c'est-à-dire définir le contenu du programme, sa répartition et sa progression didactique, ainsi que la formation des enseignants. $\mathrm{Au}$ Maroc, comme dans beaucoup d'autres pays, l'enseignement de l'informatique comme une discipline scolaire au même niveau que les mathématiques, les sciences de la vie et de la terre, la chimie et la physique peine à s'installer et cela malgré la volonté affichée et les efforts déployés par les différents intervenants dans l'éducation nationale. L'instauration d'un enseignement de l'informatique de qualité nécessite une véritable formation des enseignants. Le présent article a comme objectif de présenter un regard critique sur la formation qualifiante des enseignants d'informatique, programme, contenu pédagogique, etc., et de formuler quelques réflexions et préconisations de réforme.
\end{abstract}

\begin{abstract}
The introduction of computer science education as a science remains a hot topic in several countries. The main questions that arise each time relate to the computer science curriculum (defining the content of the program, its distribution and its didactic progression) and the teachers training. In Morocco, as in many other countries, the teaching of computer science as a school discipline at the same level as mathematics, life and earth sciences, chemistry and physics struggling to settle and this despite the will displayed and the efforts of the various stakeholders in national education. Achieving quality computer education requires real teacher training. The objective of this article is to present a critical look at the qualifying training of computer science teachers, program, educational content, etc., and to formulate some reflections and recommendations for reform.
\end{abstract}

*Corresponding author : iqdour@gmail.com 


\section{Introduction}

Dans un monde où la technologie numérique à envahit tous les domaines de l'économie à la société, il est primordial que toute personne devrait être armée avec des compétences technologiques pour pouvoir affronter un quotidien hyper technisé. De ce fait, l'école à un rôle fondamental à jouer pour réussir l'intégration des citoyennes dans l'économie et la société du savoir. Dans cette perspective, l'enseignement de l'informatique se positionne comme une composante fondamentale pour atteindre cet objectif.

En effet, à l'instar des mathématiques qui ne se limitent pas à de simples opérations de calcul ou la physique et la SVT qui sont indispensable pour faire comprendre aux jeunes le « monde naturel ». L'informatique ne consiste pas à « utiliser» une application à objectif limité, elle signifie " apprendre », " programmer » et « développer » et aussi permettre aux jeunes de s'ouvrir à un monde de plus en plus numérique.

Dans plusieurs pays, l'introduction de l'enseignement de l'informatique dans les écoles, en tant que discipline académique fait émerger plusieurs défis [1]. Parmi ces défis on peut relever la question de la formation professionnelle des enseignants [2] et la question du curricula de l'informatique c'est-à-dire quels éléments de savoir à enseigner et pour quel niveau $[3,4]$.

Le Maroc s'est engagé depuis les années 80 dans sa politique éducative à introduire un enseignement de l'informatique via plusieurs initiatives. Cependant, à partir de l'année 2000, avec l'avènement de la charte nationale de l'éducation et de la formation, qui insiste dans l'espace intitulé « Amélioration de la qualité de l'éducation et de la formation » sur la place capitale de l'utilisation des nouvelles technologies de l'information et de la communication (levier 10), que l'enseignement de l'informatique a commencé à prendre sa place dans le système éducatif [5]. C'est ainsi que l'informatique en tant que matière scolaire, au même niveau que les autres matières, avec un volume horaire précis et des objectifs bien définis fait son apparition dans l'architecture curriculaire du cursus éducatif marocain du secondaire (collégial et qualifiant) $[6,7]$.

Dans sa vision stratégique 2015-2030 élaborée à partir des recommandations du loi cadre $\mathrm{n}^{\circ}$ 51-17 relatif au système de l'éducation, de l'enseignement, de la formation et de la recherche scientifique [8] et surtout le levier 20 intitulé « Implication active dans l'économie et la société du savoir ", qui considère que les technologies d'information et de communication comme entrée dans le chantier de l'adhésion effective à l'économie et la société du savoir », le ministère de l'éducation nationale via la direction du Programme GENIE a élaboré un projet de programme d'informatique qui devrait être dispensé aux élèves de l'école primaire [9]. Ce programme qui se base sur l'enseignement de la pensée informatique [10] via l'utilisation du logiciel SCRATCH vise à développer des compétences liées à la résolution de problèmes, l'algorithmique, logique et l'abstraction à travers des exercices ou projets impliquant d'autres disciplines et, éventuellement, en profitant pour initier les élèves à d'autres aspects (comment le fonctionnement de l'ordinateur, etc.).

La mise en œuvre de l'enseignement de l'informatique nécessite une véritable formation des enseignants en nombre et en qualité. En effet, une formation de qualité des enseignants est considérée comme un paramètre clé dans l'amélioration des pratiques enseignantes et l'apprentissage des élèves et ainsi dans la réussite du système éducatif [11-13].

Dans son rapport publié en 2019 UNESCO/IFIP souligne que plusieurs pays rencontrent des difficultés pour instaurer une formation professionnalisante des professeurs d'informatique [1]. Différentes recommandations et solutions ont été proposées telles que l'encouragement des diplômés en informatique de choisir l'enseignement, ajouter une formation en informatique dans la formation initiale des professeurs du primaire, assurer une formation continue des enseignants, ....etc. 
$\mathrm{Au}$ Maroc, l'enseignement de l'informatique a été assuré, après son introduction, dans les filières technologiques ou dans les établissements où existait la discipline par des professeurs qu'on appelait "animateurs d'informatique". Mais à partir de l'année 2001/2002 et avec la vision de la généralisation de l'enseignement de l'informatique dans le système éducatif, la formation des enseignants d'informatique s'est renforcée via la création de nouveaux cadres professionnels: inspecteur d'informatique et professeur du cycle secondaire collégial chargé de l'enseignement de l'informatique au collège, l'ouverture du centre de formation des inspecteurs de l'enseignement (CFIE) et l'augmentation des effectifs des professeurs d'informatique dont la formation est effectuée dans les CPR (Centre Pédagogique Régional) et les ENS (Ecole Normale Supérieure).

Depuis 2012, une formation qualifiante des enseignants a été attribuée aux CRMEF (Centre Régional des Métiers de l'Education et de la Formation) qui ont remplacé les CPR et les CFI (Centre de formation des instituteurs) tandis que la formation initiale est assurée par les universités (aujourd'hui des établissements «Ecole Supérieure de l'Education et de la Formation » ont été créée pour prendre en charge cette mission) [14].

Après environ deux décennies de la généralisation de l'enseignement de l'informatique dans le cursus éducatif marocain, plusieurs questions restent posées sur la formation des enseignants et le contenu enseigné et son impact sur les compétences numériques acquises par les élèves. Le but principal de cet article est de dresser un aperçu critique sur la formation des enseignants d'informatique, programme et contenu tout en formulant des réflexions à partir de l'analyse des résultats d'un entretien avec les enseignants stagiaires.

\section{Formation des enseignants}

La formation des enseignants est assurée dans les CRMEF. On parle depuis 2012 de la qualification étant donné que la formation initiale devrait être faite dans les universités via des filières universitaires d'éducation (FUE). La durée de la formation dans les CRMEF était d'une année après un concours de recrutement parmi les candidats ayant une licence universitaire.

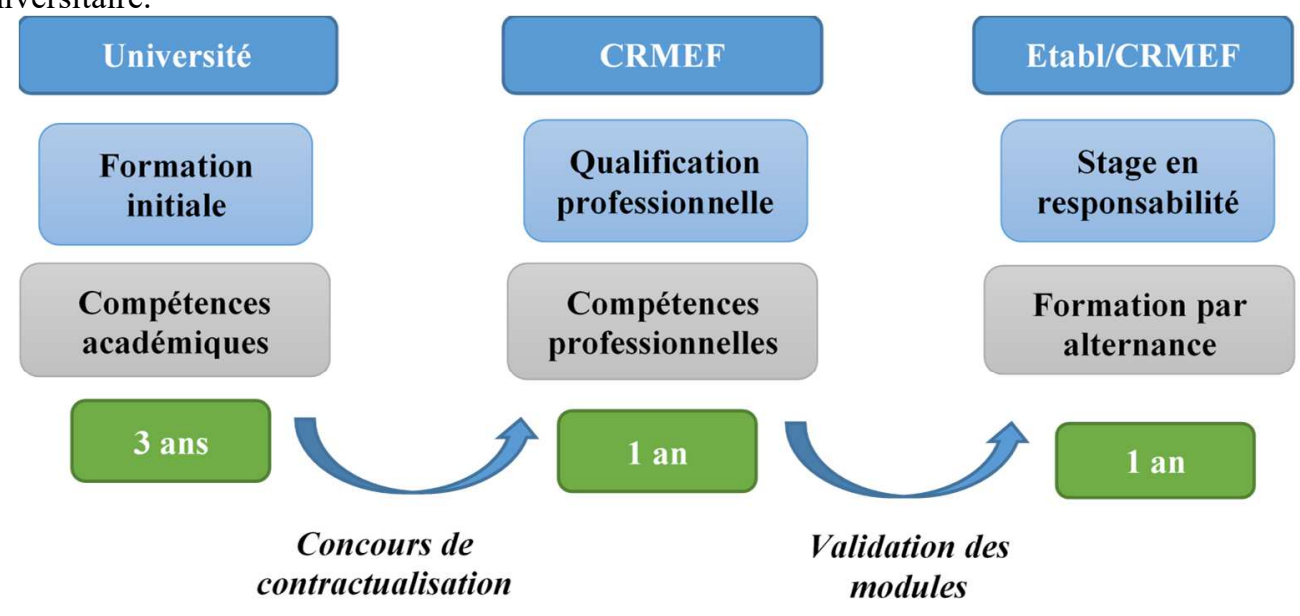

Fig.1. Processus de formation des enseignants au Maroc.

Cependant à partir de l'année 2017-2018 une nouvelle réforme de la formation des futurs enseignants a été mise en place. Cette formation devra durer cinq ans au lieu de 6 mois, voire moins pour les contractuels (durant l'année 2016/2017 le ministère par le bais des AREF a procédé à un recrutement massif des enseignants, 11000 en Janvier 2107 et 24000 en Juillet). 
Les étudiants vont suivre une formation de trois ans à l'université, sanctionnée par une licence pédagogique, avant de passer un concours pour être recrutés par les AREF et de poursuivre la formation pendant deux ans dans les CRMEF (fig.1). Durant la cinquième année, après affectation des enseignants stagiaires dans les établissements scolaires, un accompagnement et une formation pendant les vacances (60 heures) dans les CRMEF sont prévus.

Ce modèle de formation des enseignants est de type consécutif où la phase de la professionnalisation des enseignants vient après l'achèvement des études académiques c'està-dire après une formation initiale. L'autre modèle de formation est le modèle simultané, où la formation académique est combinée à la formation qualifiante et où la décision de devenir enseignant est prise plus tôt [15-17].

D'après [17] chacun de ces deux modèles présente des avantages et des inconvénients. Mais, au niveau du curriculum interdisciplinaire, où les modules éducatives et disciplinaires sont effectivement intégrés, et de la coordination entre l'enseignement universitaire et l'apprentissage pratique, le modèle simultané semble présenter certains avantages. Ceci peut expliquer pourquoi le modèle consécutif devient de plus en plus minoritaire d'après l'enquête TALIS 2018 [15].

L'architecture pédagogique du dispositif de formation dans les CRMEF est basée sur l'approche par compétences avec une architecture modulaire et l'alternance fondée sur les activités et les situations professionnelles dans les établissements scolaires afin de renforcer le caractère professionnalisant de la formation.

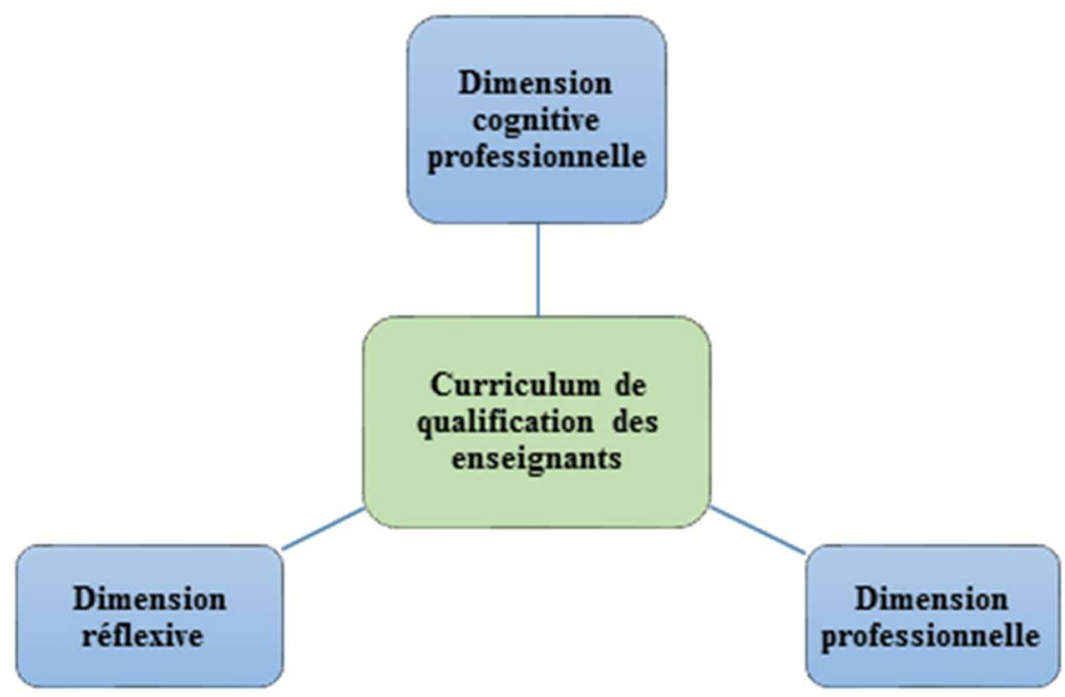

Fig.2.Articulation du curriculum de qualification des enseignants.

Le curriculum de qualification est orienté vers plus de professionnalisation. Il vise donc à former des professionnels de l'enseignement capables de réfléchir et d'agir de façon autonome et responsable. En effet, le plan de la formation a pour but de renforcer les compétences des enseignants au niveau de la discipline de spécialité et de leurs doter des compétences professionnelles. Ce plan de formation (Tableau.1) est organisé dans une architecture basée sur des modules de volume horaire fixe (30 heures).

Le choix des modules est articulé sur une vision basée sur le développement de trois dimensions (fig.2) :

- Dimension cognitive professionnelle dans lequel on trouve des modules de renforcement des acquis de base liés à la discipline de spécialité (4 modules) et le module de la science de l'éducation et celui de la didactique. 
- Dimension professionnelle qui comprend six modules : module de la planification des apprentissages, modules de la gestion des apprentissages $(60 \mathrm{~h})$, module de l'évaluation des apprentissages, module de la législation et d'éthique, module de la vie scolaire et en fin le module des TICE.

- Dimension réflexive dont l'objectif est de doter l'enseignant des compétences de réfléchir à ses pratiques et agir en suivant une démarche adéquate. Dans cette dimension on trouve le module de la méthodologie de recherche-action et deux ateliers qui concernent la production didactique et l'analyse des pratiques.

Tableau 1. Programme de formation des enseignants d'informatique.

\begin{tabular}{|c|c|}
\hline Modules (1 ${ }^{\mathrm{er}}$ semestre) & Modules ( $2^{\text {ème }}$ semestre) \\
\hline Planification des apprentissages & Gestion des apprentissages 2 \\
\hline Gestion des apprentissages 1 & Evaluation des apprentissages \\
\hline Didactique & Vie scolaire \\
\hline Science de l'éducation & Législation scolaire \\
\hline TICE & Atelier de la production didactique \\
\hline Méthodologie de recherche & Atelier de l'analyse des pratiques \\
\hline Architecture des ordinateurs & Réseaux informatiques \\
\hline Logiciels & Algorithme et programmation \\
\hline
\end{tabular}

En plus de ces modules de formation, les enseignants stagiaires sont amenés à réaliser, dans la première année, des activités pratique d'enseignement dans les établissements scolaires qui commencent par l'observation et se termine par prendre la responsabilité entière de la gestion de la classe. Dans la Deuxième année les enseignants stagiaires doivent réaliser un projet de recherche-action sous l'encadrement d'un formateur du CRMEF.

\subsection{Principaux défis}

Plusieurs défis restent à relever afin d'apporter des solutions aux problèmes de la formation des enseignants. Ainsi, malgré les efforts déployés au niveau de la formation des enseignants, les problèmes discutés dans l'article [14] après un an de l'instauration du nouveau dispositif de formation des enseignants dans les CRMEF, demeurent toujours sans solution. Ces problèmes résident dans :

- Le manque de coordination entre les universités chargées de la formation initiale et les CRMEF pour la formation qualifiante.

- Des problèmes de coordination persistent encore entre les CRMEF et les établissements scolaires attachés aux AREF ce qui nuis au principe d'une formation professionnalisante de qualité.

D'autres problèmes ont surgi avec la nouvelle conception de la formation aux CRMEF. Ces problèmes sont d'ordre organisationnel et financier surtout pour la deuxième année qui prévoit un accompagnement des nouveaux enseignants dans leurs établissements d'affectation par les formateurs et une formation présentielle durant les vacances.

\subsection{Défis de la formation des enseignants d'informatique}

Malgré l'instauration des FUE dans les universités on a constaté que plus de $66 \%$ des enseignants n'ont pas suivi cette formation. Par ailleurs, plus de $80 \%$ de ces enseignants réclament une redondance d'activités entre la formation initiale universitaire dans les FUE et la qualification professionnelle des CRMEF. 
Dans les quatre modules de renforcement des acquis de base à savoir : Architecture et fonctionnement des ordinateurs, Les logiciels, Algorithmique et Programmation et Réseaux et Internet, on trouve des savoirs et savoir-faire comme la programmation orienté objet, la sécurité des réseaux, système d'exploitation Unix etc., qui dépassent largement ce qui est réellement dispensé dans l'enseignement de l'informatique au secondaire collégial et qualifiant. Par ailleurs, les formateurs réclament que les profils différents des enseignants rendent leurs tâches difficiles de traiter tous ces concepts dans un volume horaire qui ne dépasse pas $30 \mathrm{~h}$.

Afin d'avoir une idée sur leurs regard rétrospectif critique sur la formation au CRMEF, nous avons réalisé un entretien auprès des enseignants stagiaires après un an d'expérience dans leurs établissements d'affectations. Les conclusions de cet entretien sont résumées dans les points suivants :

- Si les nouveaux enseignants admettent que la formation dans les CRMEF est ouverte à la profession, ils affirment qu'il existe un décalage entre la formation et les réalités de la profession. Ceci est en accord total avec la recherche dans ce domaine dans d'autres pays $[18,19]$.

- Les nouveaux enseignants considèrent que le stage pratique pendant la formation, suivi des deux ateliers (analyse de la pratique et production didactique) sont les modules qui contribuent le plus à leur développement professionnel.

- Malgré son importance dans le développement de la dimension réflexive des nouveaux enseignants [20], le mémoire professionnel est peu apprécié en raison de la contrainte de temps, trois mois seulement, qui coïncide avec le rituel de la rentrée scolaire. Ces difficultés démotivent les nouveaux enseignants et les empêchent de saisir le rôle important de la recherche pour réfléchir à leur pratique et développer leurs compétences.

- Concernant leurs besoins de formation continue, la gestion d'une classe qui manque de matériel pédagogique, l'évaluation des apprentissages et la gestion de la classe pour gérer les problèmes de comportement sont les points les plus récurrents.

Avec la nouvelle vision d'instaurer l'enseignement de l'informatique au primaire via l'introduction de la pensée informatique qui vise à développer chez les élèves des compétences liées à la résolution de problèmes, l'algorithmique, la logique et l'abstraction, la voie est ouverte à une réforme profonde du curriculum de l'informatique dans le cycle secondaire, en s'inspirant des recherches dans ce domaine et des expériences des pays qui ont réussi ce défi [21-24].

\section{Conclusion}

Après environ deux décennies de la généralisation de l'enseignement de l'informatique au Maroc notamment dans le cycle collégial et dans les troncs communs au cycle secondaire qualifiant et avec la réforme lancée du système éducatif via la vision stratégique 2015-2030 en plus de l'évolution technologique que connait le monde, une révision profonde du curriculum de l'informatique s'impose afin de permettre aux élèves d'intégrer une société de la communication et du savoir.

Cette réforme du curriculum doit être accompagnée d'une révision du dispositif de formation des enseignants d'informatique. Ainsi, Malgré les efforts fournis pour la professionnalisation de cette formation, certaines lacunes restent à combler comme la complémentarité entre la formation initiale et la formation professionnelle, le renforcement du stage pratique en déployant plus de ressources humaines et financières, revoir le contenu des programmes et des modules etc.... En outre, une attention particulière doit être accordée aux programmes de la formation continue des enseignants et aussi introduire dans la 
formation initiale dans les universités ou qualifiante dans les CRMEF des enseignants du primaire d'un module traitant la pensé informatique afin d'assurer la réussite de ce chantier.

\section{Références}

1. OCCE. Report of UNESCO/IFIP TC3 Meeting at OCCE, Coding Programming and the Changing Curriculum for Computing in Schools. Linz, Austria, (2018).

2. C. Angeli, J. Voogt, J. Malyn-Smith, M. Webb, A. Fluck, M. Cox, J. Zagami, The Design of the Computer Science Curriculum and the Knowledge that Teachers Need to Teach Computer Science in K-12, Education Technology and Society 19(3), 47-57 (2016).

3. M. E. Webb, Considerations for the design of Computing curricula, Paper presented at the KEYCIT - Key Competencies in Informatics and ICT, University of Potsdam, Germany, July $1-4$, (2014).

4. M. E. Webb, N. Davis, Y. J. Katz, N. Reynolds, M. M. Sysło, Towards deeper understanding of the roles of CS/ Informatics in the curriculum, Vilnius University, Lithuania. July 1-3 (2015)

5. Royaume du Maroc. Commission Spéciale Éducation Formation. Charte nationale d'éducation et de formation. (1999).

6. A. Haddi, Regards sur : Le curriculum d'informatique au cycle secondaire Marocain, Revue de l'EPI (2009).

7. L. Ouahbi, H. Darhmaoui, F. Kaddari, A. Bemmouna, A. Elachqar, A, Lahmine, Un aperçu sur l'enseignement de l'informatique au Maroc : Nécessité d'une réforme des curricula, An overview of teaching informatics in Morocco : The need for a curriculum reform, Frantice.net, 11, 51-66 (2015).

8. Royaume du Maroc. Commission Spéciale Éducation Formation. Vision stratégique, (2017).

9. MEN. Ministère de l'Éducation Nationale, de l'Enseignement Supérieur, de la Formation des Cadres et de la Recherche Scientifique, Direction GENIE. Project du programme d'informatique au cycle primaire, (2018).

10. J. M. Wing, Computational thinking, In Communications of the ACM, 49 (3), 33-35, (2006).

11. S. Guerriero. (ed.), Pedagogical Knowledge and the Changing Nature of the Teaching Profession, Educational Research and Innovation, Éditions OCDE, Paris, (2017).

12. T. Karsenti, M. Lepage, La formation pratique des enseignants. Regards croisés sur l'Afrique, l'Amérique et l'Europe (dossier thématique). Formation et Profession, 23(3), 131-133, (2015).

13. M. R. Mabejane, K. Ravanis, Linking teacher coursework training, pedagogies, methodologies and practice in schools for the undergraduate science education student teachers at the National University of Lesotho. European Journal of Alternative Education Studies, 3(2), 67-87, (2018).

14. M. Lahchimi, La réforme de la formation des enseignants au Maroc, Revue internationale d'éducation de Sèvres, 69, 21-26, (2015).

15. OCDE, TALIS 2018 Results (Volume I): Teachers and School Leaders as Lifelong Learners, OECD Publishing, (2019).

16. P. Musset, Initial teacher education and continuing training policies in a comparative perspective: Current practices in OECD countries and a literature review on potential effects, OECD, (2010). 
17. G. Ostinelli, Teacher Education in Italy, Germany, England, Sweden and Finland. European Journal of Education, Vol. 44, 2, Part II, p.291-308. Royame-Uni et États Unis : The Author Journal Compilation et Blackwell Publishing Ltd, (2009).

18. L. Darling-Hammond, K. Hammerness, P. Grossman, F. Rust, L. Shulman, The Design of Teacher Education Programs. In L. Darling-Hammond \& J. Bransford Eds), Preparing Teachers of Changing World. What teachers should Learn and Be Able to Do. San Francisco: Jossey-Bass, 390-441, (2005).

19. J. Mukamurera, C. Gingras, La formation initiale vue par des enseignants du secondaire issus des programmes de formation en cours au Québec depuis 1970, In C. Gervais \& L. Portelance (éd.), Des savoirs au cœur de la profession enseignante. Contextes de construction et modalités de partage. Sherbrooke : Editions de CRP, 45-63, (2005).

20. K. S. Volk, Action Research as a Sustainable Endeavour for Teachers: Does Initial Training Lead to Further Action? Action Research 8 (3): 315-333, (2010).

21. M. Young, Overcoming the crisis in curriculum theory: A knowledge-based approach, Journal of Curriculum Studies, 45(2), 101-118 (2013).

22. The Royal Society, Shut down or restart? The way forward for computing in UK schools, London: The Royal Society, (2012).

23. T. Plomp, R.E. Anderson, N. Law, E. Quale, Cross-National Information and Communication Technology: Policies and Practices in Education. Information Age Publishing. Charlotte. USA, (2009).

24. C. Wilson, L. A. Sudol, C. Stephenson, M. Stehlik, Running on Empty: The Failure to Teach K-12 Computer Science in the Digital Age, Association for Computing Machinery (ACM), Computer Science Teachers Association (CSTA), (2010). 